\title{
Chronic Obstructive Pulmonary Disease and Anaemia
}

\author{
Dear Editor,
}

I read the article named "The Influence of Anaemia on Mortality in Patients with Chronic Obstructive Pulmonary Disease" written by Metan et al. (1) in the last issue of the Turkish Respiratory Journal. In the study, using the hospital database the patients diagnosed to have Chronic Obstructive Pulmonary Disease (COPD) within 5-year period (2004-2008) were identified, and the prevalence of anaemia and effect of anaemia on mortality were investigated in these patients. When comparing this study with the study we carried out in our clinic on the co-occurrence of anaemia and COPD, having the same theme, and published again in the Turkish Respiratory Journal in 2012 under the topic of "Coexistence of Anaemia and COPD as a Systemic Disease" (2), the basic difference and a substantial contribution of the recent study appears to be the investigation of the effect of anaemia on mortality.

The remarkable condition in the study that would give opinion about recording system in our country is that: based on the International Statistical Classification of Diseases and Related Health Problems (ICD)-10 records, the patient files of 1791 (41.1\%) out of 4354 patients diagnosed with COPD were available, and the number of patients, whose diagnosis was confirmed by Pulmonary Function Tests (PFT), was only $511(11.7 \%)$. This, no doubt, is not under the direct responsibility of the researchers, but obviously it calls our attention to the data recording applications of the healthcare system.

Metan et al. (1) found the prevalence of anaemia higher than that found in our study (2) (32.07\% vs. 19.6\%, respectively). The most probable reason for this might be the facts that our study was prospective; previously diagnosed anaemia was an exclusion criterion in terms of inclusion-exclusion criteria, and patients with diseases that are likely to cause anaemia, such as malignancy, chronic renal insufficiency and bronchiectasis, were not included in the study. It is understood that cardiovascular diseases were the most common co-morbidities in both studies: hypertension (HT) (33.6\% vs. $25.5 \%)$, coronary artery disease (17.5\% vs. $17.6 \%)$. However, different from this study, we found the prevalence of anaemia to be significantly higher in patients with HT ( $35 \%$ vs. $5 \%, p=0.029$ ), whereas Metan et al. (1) found these two ratios to be quite close to each other (33.5\% vs. $31.3 \%)$. Considering the ratios, it is understood that this difference arises from the number of patients without HT. Both studies demonstrated that the parameters of pulmonary function test have no effect on anaemia. With regard to the stages of chronic obstructive pulmonary disease, Metan et al. (1) found the prevalence of anaemia to be $37.5 \%$ for mild-moderate COPD and $29.5 \%$ for severe-very severe COPD. The corresponding ratios were $18.3 \%$ and $21.4 \%$, respectively in our study. Although the differences regarding the presence of anaemia were not statistically significant in both two studies, the prevalence of anaemia in mild to moderate COPD patients seems to be different between the studies. The answer of the question "from where this difference arise?" may be in the percentages of patients: While the percentage of patients with mild to moderate COPD was approximately $60 \%$ in the study of Metan et al. (1), it was about $30 \%$ in our study, and cases with anaemia in this group might have had the exclusion criterion.

With regard to anaemia-mortality relationship, which was not included in our study but was the main parameter in the study performed by Metan et al. (1), it was observed that $60.7 \%$ of anaemic COPD patients died, whereas mortality rate was $43.6 \%$ for patients without anaemia $(p<0.05)$. However, as was mentioned by the researchers, mortality rate was found significantly higher $(p<0.001)$ in those with solid tumours (lung cancer accounts for the majority, $\mathrm{n}=23$ ). Mortality rate was $80.5 \%$ among 33 COPD patients with solid tumour and haematological malignancy. To what extent did the presence of this group with known effect on mortality influenced the determination that anaemia contributes to mortality in COPD patients? In the study, according to the data given in Table 1, it seems that co-morbidities (HT, coronary artery disease, diabetes mellitus, congestive heart failure, etc.) other than solid tumour had no significant relation with mortality ( $p>0.05$ ). This suggests that, aforementioned presence of solid tumour and haematological malignancy might have a significant effect on anaemia-mortality relationship. 
The facts that the prevalence of anaemia was higher than polycythemia, which is classical information, in both studies and anaemia enhances mortality while polycythemia does not, as was stated in the study conducted by Metan et al. (1) reveals the necessity of paying more attention to anaemia in chest diseases practice. It can be assumed that treatment of anaemia may decrease the mortality rates.

Ali Fidan

Department of Chest Diseases, Dr. Lütfi Kırdar Kartal Training and Research Hospital, İstanbul

Address for correspondence: Ali Fidan, Department of Chest Diseases, Dr. Lütfi Kırdar Kartal Training and Research Hospital, İstanbul, Turkey

E-mail:alifidan@yahoo.com
Received date: 28.12 .2013

Accepted date: 30.12 .2013

(c) Copyright 2014 Turkish Respiratory Society (TRS)

DOI: 10.5152/ejp.2014.46873

-Available online at www.eurasianjpulmonol.com

\section{REFERENCES}

1. Metan EÜ, Tutar N, Kanbay A, Büyükoğlan H, Oymak S, Gülmez İ, et al. The Influence of Anaemia on Mortality in Patients with Chronic Obstructive Pulmonary Disease. Solunum 2013; 15: 155-62. [CrossRef]

2. Fidan A, Tokmak T, Kıral N, Cömert SŞ, Saraç G, Salepçi S, et al. Coexistence of Anaemia and COPD as a Systemic Disease. Solunum 2012; 14 18-26. [CrossRef] 